\title{
Modeling of temperature mapping for quantitative dynamic infrared coronary angiography for intraoperative graft patency control
}

Jens Garbade, MD, Cris Ullmann, Marcus Hollenstein, Markus Johannes Barten, MD, Stephan Jacobs, MD, Stefan Dhein, MD, Thomas Walther, MD, Jan Fritz Gummert, MD, Volkmar Falk, MD, and Friedrich-Wilhelm Mohr, MD

From the Department of Cardiac Surgery, Heart Center, University of Leipzig, Leipzig, Germany.

Received for publication Sept 12, 2005; revisions received Dec 13, 2005; accepted for publication Dec 22, 2005.

Address for reprints: Jens Garbade, MD, Department of Cardiac Surgery, Heart Center Leipzig, University of Leipzig, Struempellstrasse 39, 04289 Leipzig, Germany (E-mail: jgmed93@hotmail.com).

J Thorac Cardiovasc Surg 2006;131:1344-51 $0022-5223 / \$ 32.00$

Copyright $(\odot) 2006$ by The American Association for Thoracic Surgery

doi:10.1016/j.jtcvs.2005.12.066
Objectives: Intraoperative application of thermal coronary angiography based on dynamic infrared imaging leads to useful qualitative information concerning coronary artery bypass graft flow and anatomy. Additional quantitative flow estimation is desirable to detect graft failures. The aim of this study was to develop a heat-transfer model for quantitative flow estimation in an experimental setup. The first clinical results in coronary artery bypass grafting are reported.

Methods: Dynamic infrared imaging was applied in pig hearts to collect video data of the rewarming process of the left anterior descending artery supplied by antegrade perfusion. For mathematic description, we used the dynamic enthalpy balance for open systems, and a Laplace transformation was carried out. Therefore the time constant $\tau$ was calculated by performing a nonlinear fit procedure on the averaged dynamic temperature curves recorded over a left anterior descending artery segment. Subsequently, left internal thoracic artery-left anterior descending artery bypass graft flow was assessed intraoperatively. Effective left anterior descending artery flow was determined by using a transit-time flowmeter.

Results: Tau is a system constant and changes depending on the flow and the system capacity. Assuming system capacity to be constant, $\tau$ only depends on the flow. It follows from the differential equation that there is a potential relation between $\tau$ and the flow. An excellent comparison $\left(R^{2}=0.968, P<.005\right)$ was demonstrated. By using the algorithms, quantitative flow estimation in pig hearts was possible. For clinical application, the formulas were applied to intraoperatively derived dynamic temperature curves with a good comparison to the actual left internal thoracic artery-left anterior descending artery flow.

Conclusion: The developed heat-transfer model allows for precise measurement of graft flow by using dynamic infrared imaging and can be applied for noninvasive graft flow estimation in beating-heart surgery.

$\mathrm{S}$ everal noninvasive methods, such as transit-time Doppler flow measurement (TTFM), epicardial high-frequency ultrasonography, and indocyanine green fluorescence angiography, have been used intraoperatively to assess the efficacy of coronary artery bypass grafting $(\mathrm{CABG}) .{ }^{1-3}$ Nevertheless, there is no method available to analyze qualitative and quantitative coronary blood flow simultaneously during bypass surgery. To date, angiography has been universally accepted as the gold standard for imaging the coronary arteries but is not available in most operating rooms. ${ }^{4}$

Qualitative evaluation of myocardial blood flow with epicardial temperature maps obtained by using thermal coronary angiography (TCA) was initially studied by Senyk and colleagues in $1971 .{ }^{5}$ Their results demonstrated that the detectable temperature alterations of the epicardium correlate well with the level of coronary 


\author{
Abbreviations and Acronyms \\ $\mathrm{CABG}=$ coronary artery bypass grafting \\ DIRI = dynamic infrared imaging \\ LAD $=$ left anterior descending artery \\ LITA $=$ left internal thoracic artery \\ TCA $=$ thermal coronary angiography \\ TTFM $=$ transit-time Doppler flow measurement
}

perfusion. Other studies have confirmed that there is a distinct dependence between epicardial temperature and blood flow. ${ }^{6-9}$ Robicsek and associates ${ }^{10}$ used thermography to investigate anastomotic patency and coronary anatomy, but they failed to show technical failures. With improving camera sensitivity, TCA became possible and was discussed and validated against other methods by Mohr and coworkers. ${ }^{11-13}$ A single-center study in 1401 patients ${ }^{14}$ demonstrated that TCA is a useful real-time diagnostic tool to detect possible technical failures, graft occlusions, and stenosis. The chance of immediate intraoperative revision and repair is given, which might lead to an improvement in outcome.

Some assumptions concerning qualitative myocardial blood supply and metabolic activity can be made in real time, but additional quantification of coronary flow would be highly desirable. ${ }^{11}$ Different studies have approached quantitative flow estimation, ${ }^{15-19}$ but either the dynamic character of the heat-transfer process in biologic bodies was not sufficiently implemented or technical limitations occurred. In these studies only steady-state and low flows were analyzed, limiting the ability to detect and evaluate rapid coronary flow changes. The purpose of this study was to develop a reliable heat-transfer model of the epicardial blood vessels, which start to rewarm in accordance with the amount of blood flow, to test the potential of such a model for quantification of coronary flow.

\section{Materials and Methods}

The main principle of our experimental approach was to analyze the temperature response of a homogeneous body (the heart) to the entering blood flow in the coronary tree. Assuming that the energy loss caused by convection or radiation is negligible and the temperature distribution within the coronary artery is homogeneous, a heat-transfer analysis can be applied.

\section{Heat-transfer Model}

An equation originating in the dynamic enthalpy balance for open systems was used to enable quantitative infrared flow measurements:

$$
\frac{d H}{d t}=F_{\text {in }} * T_{\text {in }}-F_{\text {out }} * T_{\text {out }}
$$

The dynamic enthalpy balance for open systems is defined as follows (potential and kinetic energy can be neglected): $\mathrm{H}$ is the
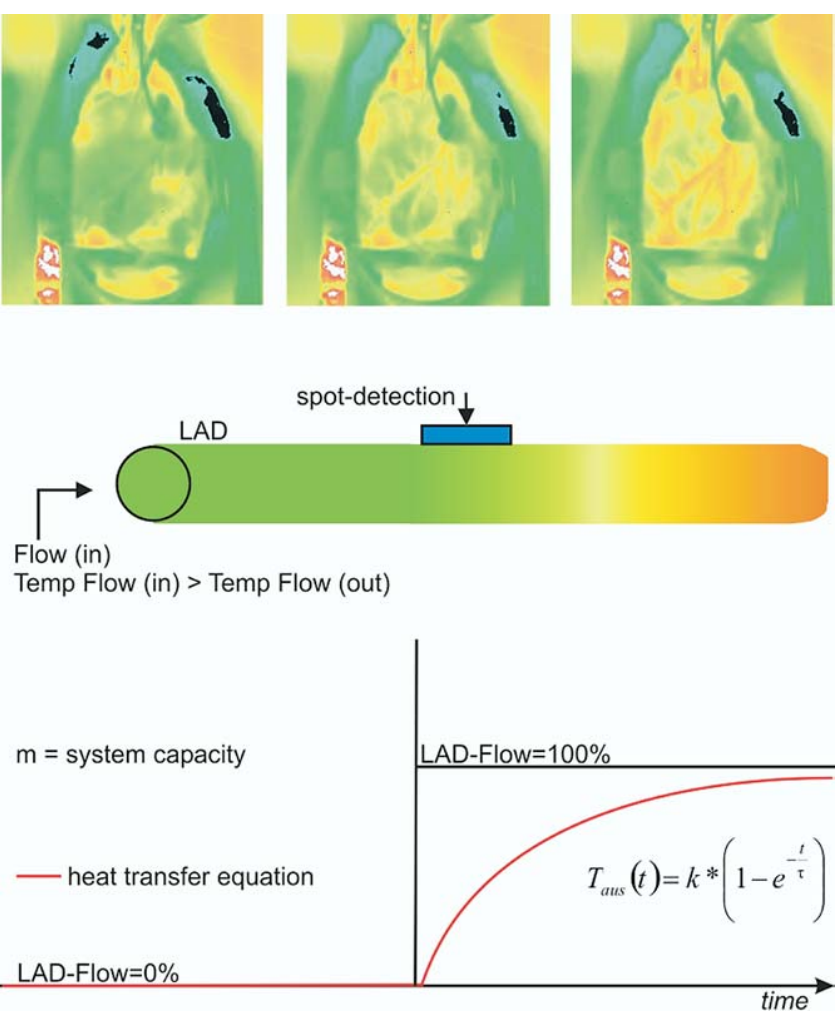

Figure 1. Dynamic infrared imaging of a pig heart with antegrade left anterior descending artery perfusion to the steady-state temperature distribution of the left anterior descending artery network. Experimental condition and mathematic description are shown. $L A D$, Left anterior descending artery.

enthalpy in kilojules, $\mathrm{T}$ is the temperature in degrees kelvin, $\mathrm{t}$ is the time in seconds, and $\mathrm{F}$ is the volume flow in milliliters per second.

After transformation of equation 1, we used equation 8 for computational and graphic $\tau$ determination:

$$
\Delta T_{\text {out }}(t)=k\left(1-e^{-\frac{t}{\tau}}\right)
$$

The complete mathematic model is described in the Appendix.

For better system modeling, a substitute system was accepted, which does not describe the process accurately but has the same dynamic behavior (Figure 1). Analytic inaccuracies are going to be eliminated by experimental adjustment of the parameters. The time constant $\tau$ is a system constant and changes depending on the flow and the system capacity. Assuming system capacity to be constant only depends on the flow. It follows from the differential equation that there is a potential relation between $\tau$ and the actual flow (see Equation 5 in the Appendix).

For the heat-transfer model, the time constant $\tau$ was calculated by performing a nonlinear fit procedure on the averaged dynamic temperature curves recorded over the spot-detected left anterior descending artery (LAD) segment for each flow. On the basis of the assumption that $\mathrm{t}$ (time) and $\tau$ are the same values from differential equation 8 , it can be concluded that $\tau$ can be graphically determined by using the $63 \%$ criterion $\left(0.63=1-e^{-1}\right)$. 


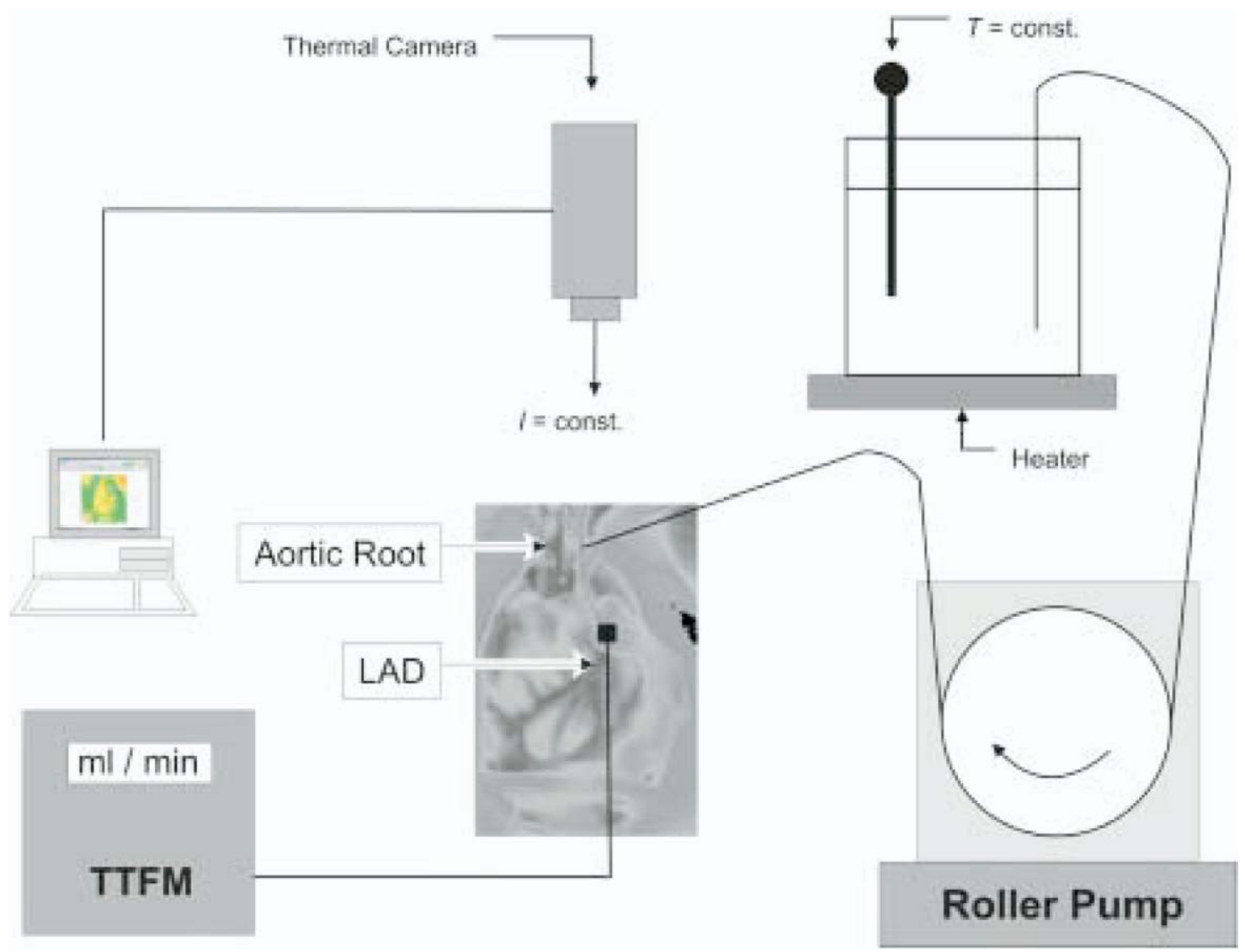

Figure 2. Experimental model. $L A D$, Left anterior descending artery; $I$, distance between dynamic infrared imaging scanner and epicardium; $T$, temperature; TTFM, transit-time Doppler flowmeter.

That means that by $63 \%$ of the maximal achieved temperature response, $\tau$ is identical to the corresponding t (time) value.

\section{Animal Experiments}

All animal procedures were approved by the Animal Care and Use Committee of the University of Leipzig and performed in accordance with the "Guide for the Care and Use of the Laboratory Animals" (1996).

Four pigs with a body weight of about $60 \mathrm{~kg}$ were used for the investigation. Premedication was performed by means of intramuscular application of atropine $(0.02 \mathrm{mg} / \mathrm{kg})$, ketamine $(10 \mathrm{mg} / \mathrm{kg})$, and azaperone $(8 \mathrm{mg} / \mathrm{kg})$. After intravenous injection of midazolam $(0.1$ $\mathrm{mg} / \mathrm{kg})$ and fentanyl $(6 \mu \mathrm{g} / \mathrm{kg})$, the pigs were orally intubated. Anesthesia was maintained with isoflurane (1.5\%-2.0\%), continuous infusion of fentanyl, and positive pressure ventilation with $80 \%$ oxygen. Standard hemodynamic monitoring during the procedure, as well as blood gas analysis, was performed. At the end of the experiment, the animals were killed by means of intravenous injection of T61. After median sternotomy, right atrial and aortic cannulation was performed, and cardiopulmonary bypass commenced. The heart was arrested with antegrade mild hypothermic cardioplegia (Bretschneider-HTK solution; Custodiol, Dr. Franz Köhler GmbH, Alsbach-Hähnlein, Germany). A roller pump (CAPS roller pump, Stoeckert, Munich) was used to quantify antegrade coronary perfusion through the aortic root. The LAD flow was confirmed by means of TTFM (Transsonic Systems Inc, Ithaca, NY). Therefore the Doppler flow probe was directly placed around the dissected LAD. Figure 2 describes the experimental setup.

\section{Clinical Validation}

The human study was reviewed and approved by the human ethics committee of the University of Leipzig. Subsequently, after informed consent, 4 patients underwent elective CABG (beating heart, $\mathrm{n}=2$; arrested heart, $\mathrm{n}=2$ ). Left internal thoracic artery (LITA)-LAD bypass graft patency and flow were assessed intraoperatively by using dynamic infrared imaging (DIRI) and the developed algorithm. In all patients LAD flow was confirmed by means of TTFM. During data acquisition, the proximal LAD segment was occluded to prevent competitive flow.

\section{Telethermometry System}

A telethermometry system with a novel, long-wave (8-10 $\mu \mathrm{m})$, narrow-band, focal plane array infrared photodetector was used. The infrared sensor consists of a $256 \times 256$-pixel array with approximately 65,000 pixels per frame that provides a temperature 
resolution of $0.006^{\circ} \mathrm{C}$ between pixels and a temporal resolution of $100 \mathrm{~Hz}$. The spatial resolution is $40 \mu \mathrm{m}$, with more than a $99.5 \%$ yield of operating pixels. The DIRI sensor was incorporated into a mobile unit composed of a camera, light-emitting diode display, and computer for initial data analysis (BioScanIR; Omnicorder Technologies, Inc, Stony Brook, NY).

The thermal sensor was placed at a distance of $1 \mathrm{~m}$ perpendicular to the surface of the target vessel, and image acquisition of 20 seconds over a spot-detected arterial segment for each flow was performed. All data were stored and analyzed by using the incorporated software (BioScanIR Analysis Program). Additional analysis was performed with statistical software (Origin 7G; Origin Lab Corp, Northampton, Mass).

\section{Results}

The temperature gradient caused by the rewarming process of the LAD by using different flow rates delineated the vascular network of the LAD in detail at all different flow rates, and in particular, low alteration of the epimyocardial temperature along the vascular structure could be demonstrated (Figure 1).

Figure 3 shows the resulting fitted exponential temperature curves from the averaged spot-detected measurements, with an excellent coefficient of determination and the calculated time constant $\tau$ for different flow rates. The time constant $\tau$ derived from the nonlinear curve fit decreased with the growing flow, and a good comparison between the coronary flow and the $\tau$ could be observed $\left(R^{2}=0.968, P<.005\right.$, Figure 4$)$.

By using the developed algorithms and the calculated correlation (Figures 3 and 4), quantitative flow assessment in the arrested pig hearts was possible, such as for flow 1 and flow 2 :

$$
\begin{aligned}
& \text { Flow } 1=165.4172 * 4.2^{-1.27008}=26.73 \mathrm{~mL} / \mathrm{min} ; \\
& \text { Flow2 }=165.4172 * 6.7^{-1.27008}=14.77 \mathrm{~mL} / \mathrm{min} .
\end{aligned}
$$

The $\tau$ value of different flows can be read from the graphic diagram by using the $63 \%$ criterion (Figure 5; $\tau$ flow $1=$ approximately 4.2 seconds, and $\tau$ flow $2=$ approximately 6.7 seconds). The synchronized reference values determined by means of TTFM were $28 \mathrm{~mL} / \mathrm{min}$ for flow 1 and $15 \mathrm{~mL} / \mathrm{min}$ for flow 2. Table 1 summarizes the computational and graphic $\tau$ values and the measured LAD flows, as determined by means of TTFM.

\section{Clinical Validation}

For the first clinical experience, the same formulas were applied to intraoperatively derived dynamic temperature curves of 4 patients. Before analysis, the epicardial surface was slightly cooled by means of application of normothermic/room temperature saline solution. Figure 6 displays the temperatureresponse curve recorded during beating-heart surgery (data from the arrested heart CABG are not shown). The BioScanIR systems' spot detection was placed on top of the LAD in the beginning of the recording; however, its distance to the LAD changed in accordance with heart motion, which is reflected in temperature alterations. Because temperature maximums were a

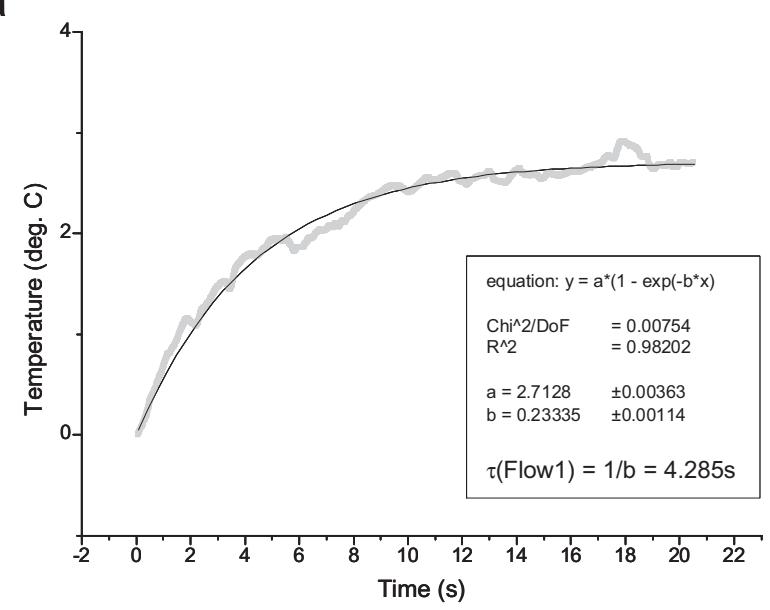

b

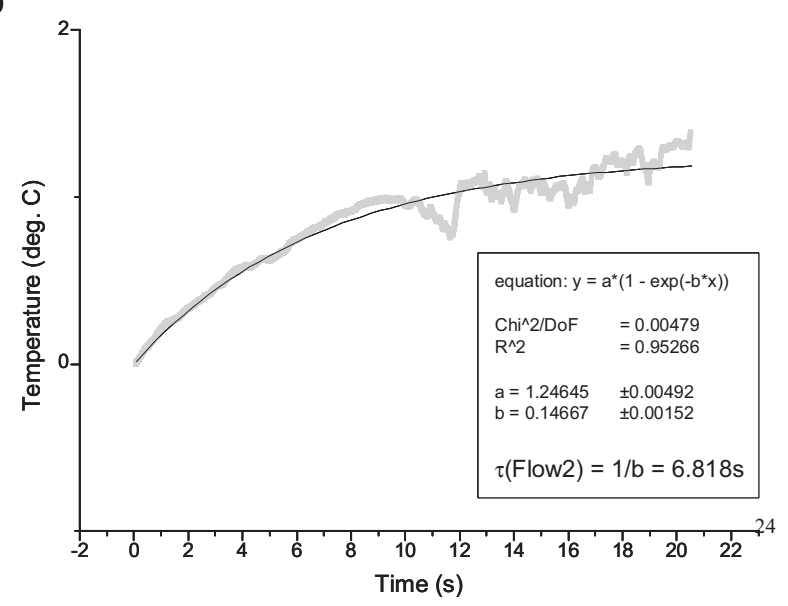

Figure 3. Curve fit for epimyocardial temperature measurements in relation to the original data points. Mathematic $\tau$ evaluation obtained for different flow rates: A, flow 1; B, flow 2.

expected while the spot detection was right above the LAD, flow estimation for that vessel requires a connection of the temperature peaks and neglecting the recorded temperature while the spot detection was not above the LAD. This is demonstrated in Figure 7, and the temperature-transfer curve could be described by using Equation 8 (Figure 7, A). For mathematic $\tau$ calculation, Figure $7, A$, was used $(\tau=6.817)$, whereas graphic $\tau$ estimation was derived from Figure 7, $B(\tau$ $=$ approximately 6.7). Table 2 shows the generated LAD flows by using DIRI and TTFM and the calculated differences between the methods.

\section{Discussion}

There is a requirement for qualitative and quantitative graft patency control to minimize complications caused by graft failure in coronary artery bypass surgery. Currently, there are 


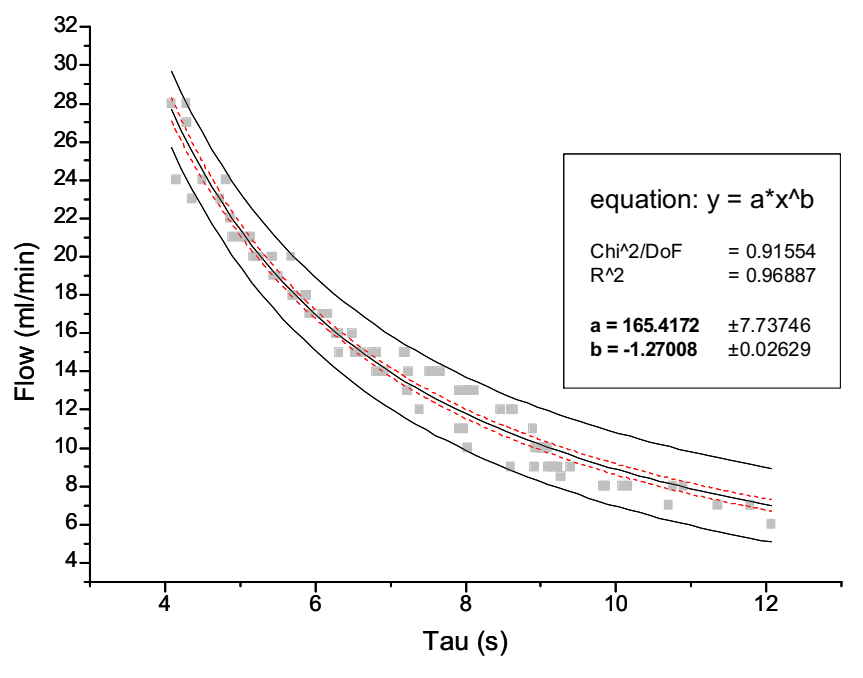

Figure 4. Comparison between different left anterior descending artery flow rates and the corresponding evaluated time constant $(\tau)$. Confidence and prediction lines are shown.

several technologies, some accepted and some evolving, that can be used in intraoperative coronary bypass graft patency control. ${ }^{20}$ Nevertheless, there is yet no method available to analyze qualitative coronary blood distribution and quantitative coronary blood flow simultaneously during bypass surgery. In this study we investigated quantitative coronary blood flow assessment by using digital infrared thermal imaging in experimental and clinical application. A specific heat-transfer algorithm was developed and applied.

It could be demonstrated that the epimyocardial temperature response, as detected with TCA, has a close dynamic correlation with actual coronary blood flow. ${ }^{5-10}$ TCA has been shown to enable an intraoperative and noninvasive evaluation

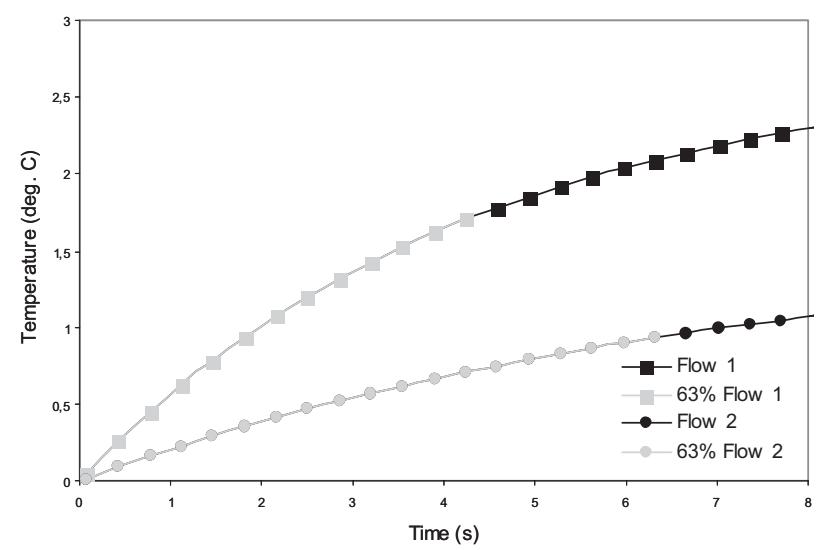

Figure 5. Curve fit for epimyocardial temperature measurements and graphic $\tau$ evaluation by using the $63 \%$ criterion for the maximal achieved temperature: flow 1 and 2.
TABLE 1. Experimental coronary flow evaluation

\begin{tabular}{lcc}
\hline & Flow 1 & Flow 2 \\
\hline Graphic $\tau$ estimation & 4.2 & 6.7 \\
Computational estimation & 4.285 & 6.818 \\
Calculated flow, mL/min & 26.73 & 14.77 \\
Gold-standard flow (TTFM), mL/min & 28 & 15 \\
Difference & $4.5 \%$ & $1.5 \%$
\end{tabular}

Experimental $\tau$ estimation with dynamic infrared imaging for 2 different flows is shown. The computational and graphic evaluated coronary blood flows are compared with the transit-time method. Differences between the investigated methods are shown. TTFM, transit-time Doppler flow measurement.

of bypass graft patency, coronary anatomy, and qualitative myocardial perfusion. ${ }^{11-13,21}$ From a recent study in 1401 patients undergoing coronary artery bypass, ${ }^{14}$ it was concluded that TCA is a reliable method to obtain real-time information on possible technical errors, such as unexpected occluding plaques or stenosis.

Different studies have approached quantitative TCA flow estimation ${ }^{15-19}$ but failed because of incomplete mathematic modeling of temperature mapping, the use of simple empiric heat-exchange models, or the limitations of older infrared systems. In this experiment an equation (Equation 1) originating in the dynamic enthalpy balance for open systems was used. This model is able to describe the temperatureanswer curve of the epicardium covering the region of the LAD. This equation differs from that being used in other studies ${ }^{16-18}$ and originating in a heat-transfer-model developed by Oster and colleagues. ${ }^{22}$ The Laplace transformation, a convenient mathematic tool to solve an ordinary differential equation, was used for further transformation (see Equations 2-8 in the Appendix). In the process of

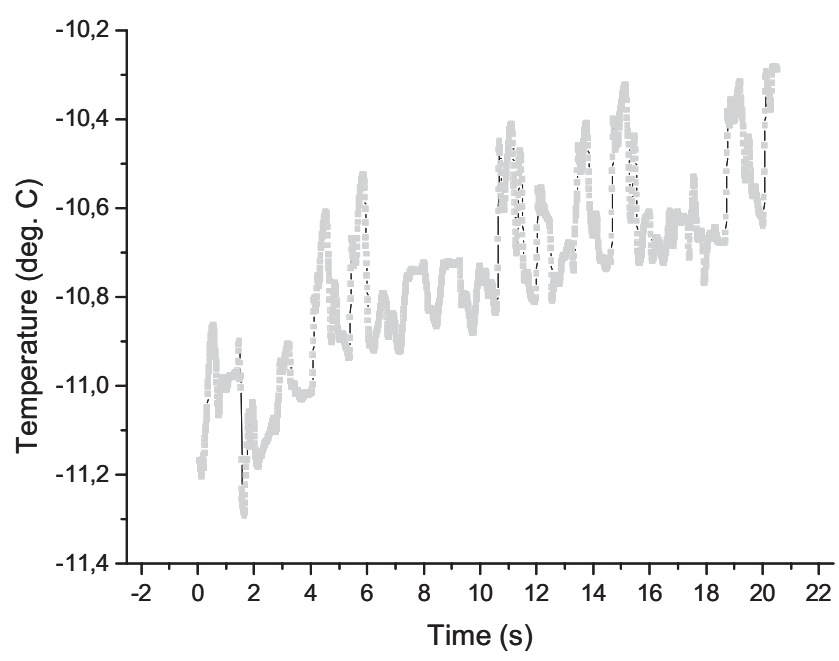

Figure 6. Epimyocardial movement of temperature response of the left anterior descending artery coronary tree in beating-heart surgery. 


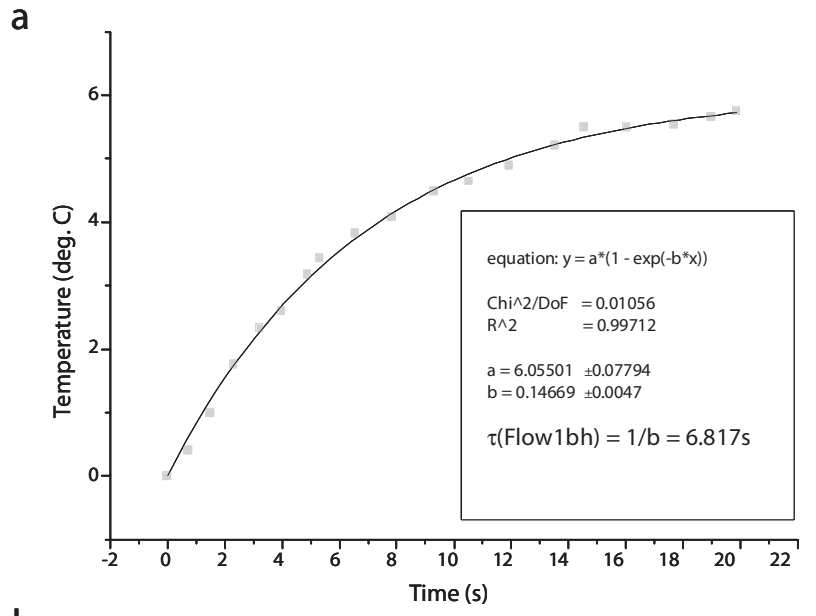

b

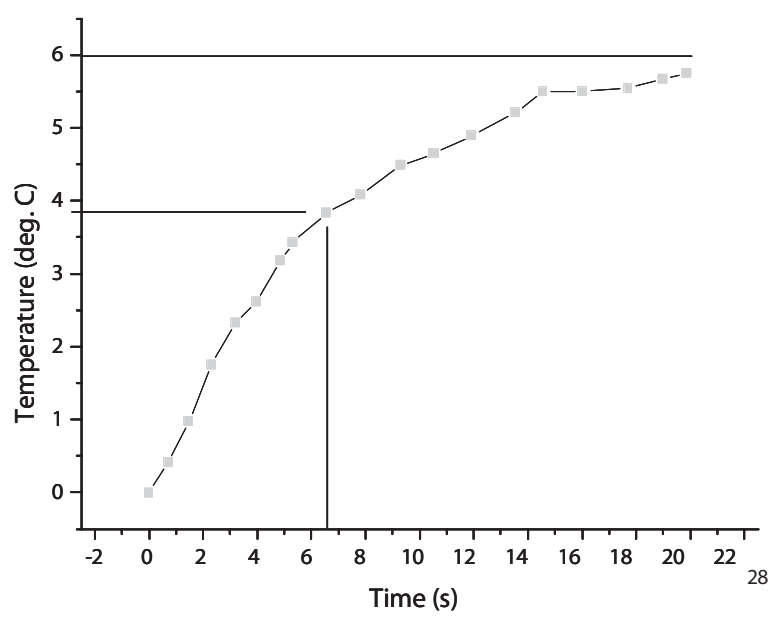

Figure 7. Curve fit and successful elimination of epimyocardial movement obtained during off-pump surgery (left internal thoracic artery-left anterior descending artery): $A$, mathematic $\tau$ evaluation; B, graphic $\tau$ evaluation.

system development, the time constant $\tau$ could be recognized as a system constant that must change in dependence of the flow and the system capacity (Figure 1). Assuming that the vessel wall condition is always the same, $\tau$ depends on the flow only. From the differential equation (see Equation 5 in the Appendix), a potential dependence of $\tau$ and flow can be concluded (Figure 4). In addition, it was shown that $\tau$ can be determined both computationally and graphically.

This article showed that potential dependence had a good coefficient of determination $\left(R^{2}=0.96887\right)$, and after calculation, an excellent correlation between $\tau$ and actual flow could be demonstrated $(r=0.984, P<.005)$. An experimental estimation of $\tau$ enabled a quantitative flow assessment in the porcine model. Intraoperatively, the dynamic behavior of the rewarming process was recorded for 20 seconds after completion of the LITA-LAD anastomosis and after declamping the
TABLE 2. First clinical results using the developed experimental approach

\begin{tabular}{lllcc}
\hline Patient & Operation & $\begin{array}{c}\text { TCA } \\
\text { estimation, } \\
\text { mL/min }\end{array}$ & $\begin{array}{c}\text { TTFM } \\
\text { estimation, } \\
\text { mL/min }\end{array}$ & Difference \\
\hline 1 & LITA-LAD & 48 & 55 & $12.7 \%$ \\
2 & Beating heart & 14.45 & 13 & $11.2 \%$ \\
3 & LITA-LAD & 17 & 20 & $15 \%$ \\
4 & Arrested heart & 18 & 20 & $10 \%$ \\
\hline
\end{tabular}

Quantitative left internal thoracic artery-left anterior descending artery flow was determined by means of thermal coronary angiography and transit-time flow measurement in beating (patients 1 and 2) and arrested (patients 3 and 4) cardiac surgery, with differences between the diagnostic methods shown. TCA, Thermal coronary angiography; TTFM, transit-time Doppler flow measurement; $L I T A$, left internal thoracic artery; $L A D$, left anterior descending artery.

bypass graft when the proximal LAD was occluded. Because of the high temperature resolution properties of $0.006^{\circ} \mathrm{C}$, an extensive cooling of the epicardium in the normothermic hearts was not necessary to achieve a temperature difference between the graft-coronary blood and the surrounding of the target coronary artery. From the achieved temperature-response curves, $\tau$ could be determined and substituted in the applied formula. The calculated blood flow was then compared with flow estimation established by using the transit-time flowmeter. Using the developed algorithm, we could demonstrate a close correlation of the temperature change along the surrounding tissue of the grafted vessel with graft flow. By analyzing the time constant $\tau$ for different flow rates, a computational, as well as graphic, assessment of actual flow in arrested and beating-heart CABG procedures were possible. After calculation, a good comparison between $\tau$ actual flows could be demonstrated.

\section{Limitations}

This study has certain limitations. The coronary arteries of the pig hearts were not diseased, and there were no atherosclerotic plaques. Furthermore, excessive epicardial fat did not exist, and coronary arteries were visible. Inaccuracies in the curve fit for low flow of less than $5 \mathrm{~mL} / \mathrm{min}$ might limit the comparison between the algorithm and the TTFM or might question the validity of the transit-time method for low flow quantification. Hol and associates ${ }^{23}$ showed that the TTFM compared with perioperative angiography could not identify significant pathologies in bypass grafts, and the interpretation of quantitative low flow measurement should be done cautiously. Additionally, TTFM and thermography are not able to identify minimal plaques in the coronary tree. This is an important limitation because flow-limiting stenosis is present only in 30\% to $40 \%$ of cases of myocardial ischemia. ${ }^{4}$

In our clinical study we only analyzed the LITA-LAD bypass grafts. It is unclear whether these results will also apply to all possible bypass configurations. In addition, an intramyo- 
cardial course of the LAD or fat tissue acts as a thermal isolator and can cause artifacts in TCA. All these factors can alter the quality of thermal angiograms and render a quantification of flow impossible. Finally, our clinical data were observed in a very small cohort because of the limited availability of the DIRI system. Therefore these clinical findings are a first step in the translation from bench to bedside, and further studies are needed to evaluate this new method in larger populations, as well as in other target vessels.

\section{Conclusion}

In summary, high-resolution real-time thermography systems combined with DIRI allow for precise delineation of coronary anatomy and possible coronary stenosis. Intraoperative application of TCA enables no-touch noninvasive information on the progress and success of an operation. Also, because of the now possible objective quantification of graft flow, technical failures can potentially be recognized more easily and prompt immediate revision.

\section{References}

1. Leong KH, Ashok V, Nishkantha A, Shan YH, Sim KW. transit-time flow measurement is essential in coronary artery bypass grafting. Ann Thorac Surg. 2005;79:854-8.

2. Balacumarasawami L, Abu-Omar Y, Choudhary B, Pigott D, Taggart DP. a comparison of transit-time flowmetry and intraoperative fluorescence imaging for assessing coronary artery bypass graft patency. J Thorac Cardiovasc Surg. 2005;130:315-20.

3. Detter C, Russ D, Iffland A, et al. Near-infrared fluorescence coronary angiography: a new noninvasive technology for intraoperative graft patency control. Heart Surg Forum. 2002;5:364-9.

4. Rudd JH, Davies JR, Weissberg PL. Imaging of atherosclerosis-can we predict plaque rupture? Trends Cardiovasc Med. 2005;15:17-24.

5. Senyk J, Malm A, Bornmyr S. Intraoperative cardiothermography. A new method for detecting ischemic areas in the heart muscle and for investigating the results of revascularization procedures in coronary surgery. Eur Surg Res. 1971;3:1-12.

6. Kekesi V, Papp L, Juhasz-Nagy A. Haemodynamic and thermographic signs of intramyocardial venous outflow redistribution induced by coronary sinus occlusion in the canine heart. Acta Chir Hung. 1986;27:203-15.

7. Papp L, Allo G, Szabo Z, Juhasz-Nagy A. Natural history of acute regional myocardial ischaemia revealed by infrared thermography in the canine heart. Acta Morphol Hung. 1985;33:123-42.

8. Papp L, Mezei B, Osvath B, Szabo Z. Thermography in artificially induced coronary circulatory disorders. Acta Chir Acad Sci Hung. $1982 ; 23: 75-81$

9. Rogowski J, Mrozinski P, Jagielak D, Lango R, Narkiewicz M, Wujtewicz M. Thermographic assessment of stellate ganglion block effectiveness during cardiosurgical procedures. Med Sci Monit. 2000;6:407-10.

10. Robicsek F, Master TN, Svenson RH, et al. The application of thermography in the study of coronary blood flow. Surgery. 1978;84:858-64.

11. Mohr FW, Matloff J, Grundfest W, et al. Thermal coronary angiography: a method for assessing graft patency and coronary anatomy in coronary bypass surgery. Ann Thorac Surg. 1989;47:441-9.

12. Mohr FW, Falk V, Krieger H, et al. IMA-graft patency control by thermal coronary angiography during coronary bypass surgery. Eur J Cardiothorac Surg. 1991;5:534-41.

13. Mohr FW, Grundfest W, Litvack F, Forrster J, Matloff J, Kirchhoff PG. Digital thermo-coronary angiography-development and validation of the method in comparison with conventional cine-coronary angiography. Z Kardiol. 1989;78:441-52.

14. Sönmez B, Arbatli H, Tansal S, et al. Real-time patency control with thermal coronary angiography in 1401 coronary artery bypass grafting patients. Eur J Cardiothorac Surg. 2003;24:961-6.
15. Adachi H, Becker LC, Ambrosio G, et al. Assessment of myocardial blood flow by real-time infrared imaging. J Surg Res. 1987;43:94-102.

16. Falk V, Walther T, Kitzinger H, et al. An experimental approach to quantitative thermal coronary angiography. Thorac Cardiovasc Surg. 1998;46:25-7.

17. Gordon N, Rispler S, Sideman S, Shofti R, Beyar R. Estimation of coronary blood flow by ECG gated cardiac thermography in openchest conditions. Physiol Meas. 1998;19:353-66.

18. Gordon N, Rispler S, Sideman S, Shofti R, Beyar R. Thermographic imaging in the beating heart: a method for coronary flow estimation based on a heat transfer model (F). Med Eng Phys. 1998;20:443-51.

19. Papp L, Allo G, Kekesi V, Szabo Z, Juhasz-Nagy A. Computer-aided thermographic evaluation of disturbances of the coronary adaptive reserve: an experimental study. Acta Morphol Hung. 1985;33:179-88.

20. Wolf RK, Falk V. Intraoperative assessment of coronary artery bypass grafts. J Thorac Cardiovasc Surg. 2003;126:634-7.

21. Falk V, Walther T, Philippi A, et al. Thermal coronary angiography for intraoperative patency control of arterial and saphenous vein coronary artery bypass grafts: results in 370 patients. J Card Surg. 1995;10:147-60.

22. Oster H, Schollhorn J, Zuchner K, Leitz KH. Thermographic evaluation of myocardial temperature during infusion of cold cardioplegia. Thorac Cardiovasc Surg. 1983;31:31-4.

23. Hol PK, Fosse E, Lundblad R, et al. Graft control by transit-time flow measurement and intraoperative angiography in coronary artery bypass surgery. Heart Surg Forum. 2002;4:254-8.

\section{Appendix}

The basis of the system is the dynamic enthalpy balance for open systems (Equation 1).

After transformation of Equation 1, we receive the following equation for further treatment:

$$
\frac{m}{F_{\text {in }}} * \frac{d \Delta T_{\text {out }}(t)}{d t}+\Delta T_{\text {out }}(t)=\Delta T_{\text {in }}(t)
$$

Function 2 can now be transferred to the s domain by means of Laplace transformation. The main use of the Laplace transformation is to study the transients of the signals. Any function, $\mathrm{f}(\mathrm{t})$, can be regarded as a signal, which is a graph in the time domain. The Laplace transformation transforms the time domain function to a function in the s domain, which is called the frequency domain. Hence this is a convenient tool to transform a problem in the time domain to a frequency domain and vice versa. That is the practical application of the Laplace transformation. Mathematically, it is a tool to solve an ordinary differential equation.

Laplace transformation in the s domain (frequency domain) is defined as follows:

$$
\begin{gathered}
\frac{m}{F_{\text {in }}} * s \Delta T_{\text {out }}(s)+\Delta T_{\text {out }}(s)=\Delta T_{\text {in }}(s) \\
\text { Transfer function }: \frac{\text { Out }}{\text { In }}=\frac{\Delta T_{\text {out }}}{\Delta T_{\text {in }}} \\
\left(\frac{m}{F_{\text {in }}} s+1\right) \Delta T_{\text {out }}=\Delta T_{\text {in }} \Rightarrow \frac{\Delta T_{\text {out }}}{\Delta T_{\text {in }}}=\frac{1}{\left(\frac{m}{F_{\text {out }}} * s+1\right)}
\end{gathered}
$$




$$
\tau=\frac{m}{F_{\text {in }}}
$$

Unit jump in the Laplace domain is defined as follows: $\frac{1}{s}$.

In this case, however, there is no unit jump but a jump with the intensification $k$, and therefore we get $\frac{k}{s}$.

$$
T_{\text {out }}=\frac{1}{\left(\frac{m}{F_{\text {in }}} * s+1\right)} * \frac{k}{s}
$$

Finally, a transformation from the s domain back to the time domain was performed:

$$
T_{\text {out }}(t)=k\left(1-e^{-\frac{t}{\tau}}\right)+T_{\text {in }}(t=0)
$$

A description of the temperature-response curve with equation 7 is reasonable and enables a $\tau$ estimation by means of calculation. Calculation of $\tau$ for each flow by using the developed mathematic algorithm is performed as follows:

$$
\Delta T_{\text {out }}(t)=k\left(1-e^{\left.-\frac{t}{\tau}\right)}\right.
$$

